\title{
artigo
}

Moraes, J.T.; Assis, I.I.C.; Nunes, V.C.; Faria, R.G.S.; Rodrigues, M.O.; Sampaio, L.R.L.

Perfil de idosos com estomias em uma região de Minas Gerais

\section{Perfil de idosos com estomias em uma região de Minas Gerais}

\author{
Profile of elderly with stomas in a region of Minas Gerais \\ Perfil de ancianos con estomas en una región de Minas Gerais
}

\begin{abstract}
RESUMO
Objetivo: Descrever o perfil de pessoas idosas com estomias de uma microrregião de saúde do Centro-Oeste mineiro. Método: Estudo descritivo, transversal e documental realizado por meio da análise de prontuários utilizados para cadastro de pessoas com estomias. Resultados: A amostra foi composta por 85 idosos, com idade média de 73 anos, prevalência do sexo feminino (55,3\%), casados (55,3\%), ensino fundamental completo ou não $(78,8 \%) ; 75,3 \%$ eram aposentados e com renda de até dois salários mínimos $(87 \%)$. Observou-se predominância do tipo colostomia $(72,9 \%)$ em caráter definitivo $(76,5 \%)$, por câncer $(84,7 \%)$ e confeccionada há mais de 3 anos $(57 \%)$. Tendo como dependência total ou parcial $(49,4 \%)$ para a realização do autocuidado cotidiano. Conclusão: Observou-se que os idosos com estomias de um SASPO de uma microrregião de saúde do Centro Oeste Mineiro eram, em sua maioria, do sexo feminino, casados, com baixo grau de instrução, aposentados e com renda igual a dois salários mínimos, com predominância de estomia do tipo colostomia, definitiva, por câncer e confeccionada há mais de 3 anos. Foi possível conhecer o perfil dos idosos que convivem com a estomia, contribuindo para que os profissionais de saúde possam planejar sua assistência buscando proporcionar melhor qualidade de vida aos idosos com estomia.
\end{abstract}

DESCRITORES: Estomia, Idoso, Perfil de Saúde.

\section{ABSTRACT}

Objective: To describe the profile of elderly people with ostomy in a health micro-region in the Midwest of Minas Gerais. Method: Descriptive, cross-sectional and documentary study conducted through the analysis of medical records used to register people with stomas. Results: The sample consisted of 85 elderly people, with an average age of 73 years, prevalence of females $(55.3 \%)$, married (55.3\%), complete elementary school or not $(78.8 \%) ; 75.3 \%$ were retired and with an income of up to two minimum wages $(87 \%)$. There was a predominance of the colostomy type $(72.9 \%)$ in a definitive character $(76.5 \%)$, due to cancer $(84.7 \%)$ and made over 3 years ago $(57 \%)$. Total or partial dependence $(49.4 \%)$ for daily self-care. Conclusion: It was observed that the elderly with ostomy from a SASPO in a health micro-region in the Midwest of Minas Gerais were mostly female, married, with low education, retired and with an income equal to two minimum wages, with a predominance of colostomy-type ostomy, definitive, due to cancer and made more than 3 years ago. It was possible to know the profile of the elderly who live with the ostomy, contributing so that health professionals can plan their assistance seeking to provide a better quality of life to the elderly with an ostomy.

DESCRIPTORS: Ostomy, Elderly, Health Profile.

\section{RESUMEN}

Objetivo: Describir el perfil de ancianos con ostomía en una microrregión de salud del Medio Oeste de Minas Gerais. Método: Estudio descriptivo, transversal y documental realizado mediante el análisis de historias clínicas utilizadas para el registro de personas con estomas. Resultados: La muestra estuvo conformada por 85 ancianos, con edad promedio de 73 años, prevalencia femenina $(55,3 \%)$, casados (55,3\%), primaria completa o no (78,8\%); El 75,3\% estaban jubilados y con un ingreso de hasta dos salarios mínimos (87\%). Predominó el tipo de colostomía $(72,9 \%)$ en carácter definitivo $(76,5 \%)$, por cáncer $(84,7 \%)$ y realizada hace más de 3 años (57\%). Dependencia total o parcial (49,4\%) para el autocuidado diario. Conclusión: Se observó que los ancianos con ostomía de un SASPO de una microrregión de salud en el Medio Oeste de Minas Gerais eran en su mayoría mujeres, casados, con baja educación, jubilados y con un ingreso igual a dos salarios mínimos, con predominio de ostomías tipo colostomía, definitivas, por cáncer y realizadas hace más de 3 años. Se pudo conocer el perfil de los ancianos que conviven con la ostomía, contribuyendo a que los profesionales de la salud puedan planificar su asistencia buscando brindar una mejor calidad de vida a los ancianos con ostomía.

DESCRIPTORES: Estomía, Anciano, Perfil de Salud.

RECEBIDO EM: 13/10/2020 APROVADO EM: 29/10/2020 


\section{Juliano Teixeira Moraes}

Enfermeiro Estomaterapeuta, Doutor. Professor Adjunto do Curso de Enfermagem - Universidade Federal de São João del-Rei - Campus Centro Oeste, Divinópolis, Minas Gerais, Brasil.

ORCID: 0000-0002-1109-962X

\section{Itaricely Istolé Castro de Assis}

Acadêmica do curso de Enfermagem da Universidade Federal de São João del-Rei - Campus Centro Oeste, Divinópolis, Minas Gerais, Brasil.

ORCID: 0000-0002-8430-5869

\section{Victória Corrêa Nunes}

Acadêmica do curso de Enfermagem da Universidade Federal de São João del-Rei -- Campus Centro Oeste, Divinópolis, Minas Gerais, Brasil.

ORCID: 0000-0002-9751-2031

\section{Rafaela das Graças Santiago Faria}

Enfermeira. Mestranda em Enfermagem pela Universidade Federal de São João del-Rei - Campus Centro Oeste, Divinópolis, Minas Gerais, Brasil.

ORCID: 0000-0001-9508-8372

\section{Mariella Oliveira Rodrigues}

Enfermeira da Estratégia da Saúde da Família da cidade de Divinópolis, Minas Gerais, Brasil.

ORCID: 0000-0002-5706-9258

\section{Luis Rafael Leite Sampaio}

Enfermeiro Estomaterapeuta, Doutor. Universidade Regional do Cariri, Ceará, Brasil.

ORCID: 0000-0003-1437-9421

\section{INTRODUÇÃO}

0 desenvolvimento da longevidade das pessoas no mundo tem sido proporcionado pelo avanço das pesquisas e tecnologias. No Brasil, estima-se que a população de idosos no ano de 2025 atinja a margem de 34 milhões, o que equivale $15 \%$ da população ${ }^{6}$. É considerável que o aumento da expectativa de vida possibilita acompanhar melhor os parâmetros de saúde das populações ${ }^{16}$.

Assim, as Doenças Crônicas Não Transmissíveis (DCNT's), tem se destacado nas últimas décadas em consequência do maior número de casos de patologias como diabetes, câncer, doenças do aparelho circulatório e doenças respiratórias crônica ${ }^{8}$.

Nesse sentido, um dos diagnósticos que predominam a tomada de decisão para se realizar uma confecção de estomia intestinal em idosos, seria o câncer, especificamente de colorretal ${ }^{5,9}$.

O procedimento cirúrgico no qual é utilizado para tratamento, consiste em
Nesse sentido, um

dos diagnósticos que predominam a

tomada de decisão

para se realizar uma

confecção de estomia

intestinal em idosos,

seria o câncer,

especificamente

de colorretal uma abertura para exteriorização da luz de um órgão, criando uma comunicação com o meio externo ${ }^{6}$. As estomias podem ser respiratórias, de alimentação ou de eliminação, temporárias ou permanentes. As de eliminação são as mais comuns sendo elas as colostomias, ileostomias e urostomias ${ }^{3}$.

Segundo a Ostomy Associations of America (UOAA), o número de estomias ascenderam. 150.00 americanos possuem uma estomia e são realizadas 130.000 cirurgias de confecção ao ano. No Brasil a estatística anual é de aproximadamente um milhão e 400 mil procedimentos cirúrgicos $^{5}$. Dados que podem estar acompanhando a atual dinâmica demográfica mundial, com o avanço no processo de envelhecimento populacional ${ }^{1}$.

Sabe-se que a construção da estomia pode aumentar a expectativa de vida das pessoas e ajuda-las a retornar com suas atividades de vida diária. Todavia, esse processo pode gerar impactos no indivíduo em âmbitos fisiológicos, psicológicos e sociais ${ }^{2}$.

Conhecer o perfil desta população for- 
nece informações e subsídios que contribuem para construção de políticas de saúde, gestão e dados para o planejamento da assistência à saúde das pessoas idosas com estomias onde proporcione reabilitação e melhor qualidade de vida. Portanto, foi objetivo deste estudo conhecer e descrever o perfil de pessoas idosas com estomias de uma microrregião de saúde do Centro-Oeste mineiro.

\section{MÉTODO}

Trata-se de um estudo descritivo, transversal e documental realizado por meio da análise de informações registradas em prontuários utilizadas para cadastro de pessoas com estomias atendidas e acompanhadas no Serviço de Atenção à Saúde da Pessoa com Estomias (SASPO), em uma microrregião de saúde do Centro-Oeste mineiro.

O SASPO é referência para 13 municípios e compreende uma extensão territorial de $6.601,7 \mathrm{Km} 2$, com uma população estimada em 433.163 habitantes. É um serviço que possui uma equipe multidisciplinar, composta por médico, enfermeiro, assistente social, psicólogo e nutricionista. Presta assistência especializada, ações de reabilitação, incluindo orientação para o autocuidado, prevenção, tratamento de complicações, capacitação e fornecimento de equipamentos coletores e adjuvantes.

Todos os prontuários do serviço possuem ficha cadastral que compreende a

Tabela 1. Perfil Sociodemográfico e econômico das pessoas idosas com estomias registradas no Serviço de Atenção à Saúde das Pessoas com Estomias em uma micro região de saúde do Centro-Oeste mineiro, 2018.

\section{Dados sociodemográficos e econômicos}

Variáveis

N

$\%$

Idade

60 a 70 anos

37

43,5

71 a 80 anos

29

34,1

$>81$ anos

19

22,3

\section{Sexo}

Masculino

38

44,7

Feminino

47

55,3

Estado civil

avaliação de enfermagem e médica no momento de adesão ao sistema. Destes, foram incluídos todos os prontuários de usuários com registro ativo no SASPO desde a sua implantação em maio de 2011 que tinham idade maior ou igual a 60 anos completos no dia da coleta de dados. Perfazendo um total de 85 prontuários de pessoas idosas incluídos nesta investigação. Foram excluídos 37 prontuários de usuários que se desligaram do serviço por motivo de óbito, reversão cirúrgica do estoma ou abandono do tratamento.

A coleta de dados aconteceu no período de janeiro a março de 2018. Foram registradas as seguintes variáveis de estudo: idade, sexo, estado civil, grau de instrução, situação de trabalho, renda familiar, tipo de estomia, permanência da estomia, diagnóstico, comorbidades, data da cirurgia e avaliação para o autocuidado.

Os dados obtidos foram armazenados em um banco de dados utilizando-se uma planilha do programa Microsoft Excel $^{\circledR}$ versão 2016, de forma a possibilitar a análise estatística dos dados, os quais estão apresentados em forma de tabelas e gráficos. Foram realizadas análise descritiva das variáveis de caracterização amostral.

$\mathrm{O}$ estudo respeitou os preceitos éticos determinados pela Resolução CONEP $n^{\circ} 466 / 2012$ e foi aprovado pelo Comitê de Ética em Pesquisa com seres humanos da Universidade Federal de São João del-Rei/Campus Centro Oeste por meio do parecer n. n. 714.992 (CAAE: 32873614.0.0000.5545). Por se tratar de dados secundários, não foi aplicado o Termo de Consentimento Livre e Esclarecido, já que não houve contato ou outra forma de comunicação com as pessoas envolvidas na pesquisa.

\section{RESULTADOS}

Foi possível identificar 85 prontuários de pessoas idosas com estomias que correspondia a $58 \%$ da população de pessoas com estomias registradas nesse SASPO. Esse grupo de pessoas idosas com estomias possuía idade média de 73 $(\mathrm{dp}=8,18)$ anos, sexo feminino $(55,3 \%)$, 


\begin{tabular}{|c|c|c|}
\hline Solteiro & 9 & 10,6 \\
\hline Casado & 47 & 55,3 \\
\hline Viúvo & 24 & 28,2 \\
\hline Divorciado & 5 & 5,9 \\
\hline \multicolumn{3}{|l|}{ Escolaridade } \\
\hline Nenhuma & 6 & 7,1 \\
\hline Fundamental completo & 39 & 45,9 \\
\hline Fundamental incompleto & 28 & 32,9 \\
\hline Ensino médio completo & 7 & 8,2 \\
\hline Ensino médio incompleto & 1 & 1,2 \\
\hline Superior completo & 4 & 4,7 \\
\hline \multicolumn{3}{|l|}{ Situação de trabalho } \\
\hline Trabalho informal & 5 & 5,9 \\
\hline Estudante & 2 & 2,4 \\
\hline Dona de casa & 12 & 14,1 \\
\hline Aposentado & 64 & 75,3 \\
\hline Afastado & 2 & 2,4 \\
\hline \multicolumn{3}{|l|}{ Renda } \\
\hline < 1 salário & 11 & 12,9 \\
\hline 1 salário & 5 & 5,9 \\
\hline 2 salários & 58 & 68,2 \\
\hline 3 salários & 9 & 10,6 \\
\hline 4 salários & 1 & 1,2 \\
\hline 5 salários & 1 & 1,2 \\
\hline Fonte: SASPO (2018) & & \\
\hline
\end{tabular}

Tabela 2: Perfil clínico das pessoas idosas com estomias registradas no Serviço de Atenção à Saúde das Pessoas com Estomias em uma micro região de saúde do Centro-Oeste mineiro, 2018.

\section{Variáveis}

N

$\%$

\section{Tipo de Estomia}

lleostomia

2

2,4

Colostomia

62

72,9

Urostomia

Permanência do Estoma

Temporária

20

23,5

Definitiva

Diagnósticos

Câncer casados (55,3\%) e em relação ao nível de escolaridade, a maioria possuía ensino fundamental completo $(45,9 \%)$ e ensino fundamental incompleto $(32,9 \%)$. Também foi identificado que $75,3 \%$ deles eram aposentados e possuíam uma renda de até dois salários mínimos (87\%) (Tabela 1).

Quanto ao perfil das estomias e dados clínicos, observou-se predominância de estomia do tipo colostomia (72,9\%), em caráter definitivo $(76,5 \%)$, tendo como principal causa o diagnóstico de câncer $(84,7 \%)$ e confeccionada há mais de 3 anos (57\%). Cabe destacar que 49,4\% deles registraram dependência total ou parcial para a realização dos cuidados para o autocuidado cotidiano (Tabela 2).

\section{DISCUSSÃO}

Estudos tem evidenciado que a incidência de câncer tem acompanhado o crescimento do envelhecimento populacional14. Para o Brasil, o Instituto Nacional de Câncer José Alencar Gomes da Silva (INCA) estima que a cada ano do triênio 2020-2022 ocorrerão 625 mil casos novos de câncer e 41 mil serão de câncer cólon e reto; 20.520 casos de câncer de cólon e reto serão em homens e 20.470 em mulheres. Estatisticamente pode corresponder a um risco estimado de 19,63 casos novos a cada 100 mil homens e 19,03 para cada 100 mil mulheres ${ }^{7}$.

Sabe-se que no país, indivíduos idosos são em sua maioria mulheres e este processo se denomina feminização do envelhecimento populacional. As mulheres representam o maior número da amostra, o que justifica que buscam mais por assistência médica para patologias como câncer, que podem gerar confecção de estomas, considerando que os homens recorrem em menor frequência aos serviços de saúde para prevenção de $\operatorname{agravos}^{10}$.

Considerando o estado civil, com destaque para pessoas casadas, questóes relativas ao companheirismo e sexualidade, são importantes. Com o envelhecimento, mesmo sem uma estomia, pode ocorrer o cessar da prática ou diminuição do ato sexual, isto se deve a disfunção fisiológica, 
Doença Inflamatória Intestinal

Chagas

Outras

Data da cirurgia

$<1$ Ano

1 A a 3 Anos

$3 \mathrm{~A}$ a 5 Anos

$5 \mathrm{~A}$ a10 Anos

$>10$ Anos

Avaliação para 0 autocuidado

Apto

Parcialmente dependente

Dependente

Fonte: SASPO (2018) como a perda da libido causadas pela distorção da imagem corporal $l^{4}$.

No entanto, a relação afetiva do cônjuge é essencial para construção da rede de reabilitação. O companheirismo contribui de forma benéfica a superação de momentos difíceis inerentes à doença $\mathrm{e}$ estomia ${ }^{13}$.

A baixa escolaridade pode interferir no processo de cuidar e gerar baixa adesão no aprendizado de autocuidado da pessoa com estomia em qualquer idade ${ }^{10}$. Assim, considerando este grupo de idosos, o fato de possuírem no máximo o ensino fundamental em sua maioria, faz com que os profissionais da saúde adaptem suas condutas de orientação e educação em saúde para o autocuidado ${ }^{11}$.

Além disto, a baixa escolaridade interfere nas ocupações trabalhistas que requerem pouca qualificação. A maioria dos idosos com estomia do estudo eram aposentados e sobreviviam com renda familiar de até dois salários mínimos. Embora a assistência à saúde da pessoa com estomias esteja assegurada pelo Sistema Único de Saúde (SUS), eles se veem em alguns momentos fragilizados e necessitando de cuidados especializados, tendo que arcar com as despesas de equipamentos coletores,

\section{Além disto, a baixa}

escolaridade interfere

\section{nas ocupações}

trabalhistas que

requerem pouca

qualificação. A

maioria dos idosos

com estomia

do estudo eram

aposentados e

sobreviviam com

renda familiar de até

dois salários mínimos. consultas ou até mesmo medicamentos, no sistema privado?.

Em relação ao tipo de estomia, a colostomia com tempo de permanência definitiva se destacou e tendo em vista que o maior grupo é de idosos aptos para o autocuidado, as técnicas de irrigação, alimentação e higiene proporcionaram mais autonomia. Além de que, as pessoas com colostomia têm ainda mais facilidade para realizarem tais procedimentos e utilizar outros dispositivos, como de cobertura do estoma, coletores que inibem ruídos, dentre outros ${ }^{12}$.

O fato dos idosos terem apresentado estomias irreversíveis pode estar diretamente relacionado a fragilidade do idoso e questões de complicações interligadas com a idade avançada $1^{7}$. No que tange à assistência a pessoas idosas com estomias, os profissionais de saúde, especialmente enfermeiros, devem considerar as questões inerentes à fragilidade do ser humano idoso. Além disto, há possibilidade do idoso com estomia enfrentar outras situações de doença como a depressão, ansiedade, alterações de imagem corporal, problemas sexuais e solidão ${ }^{2}$.

O idoso com estomia, é cercado de inseguranças e sofre constantemente com condições limitantes como a diminuição de capacidade corporal, além de que, em dado momento lhe é imposto responsabilidades de autocuidado e manutenção de um dispositivo que irá acompanha-lo temporariamente ou definitivamente ${ }^{15}$.

Há de se considerar que apesar desta investigação trazer como limitação o tamanho da população, atingiu-se o objetivo de definir o perfil de pessoas idosas com estomias de uma microrregião de saúde do Centro-Oeste mineiro.

\section{CONCLUSÃO}

A partir da análise dos prontuários dos participantes deste estudo, pode-se observar que os idosos com estomias de um SASPO de uma microrregião de saúde do Centro Oeste mineiro eram, em sua maioria, do sexo feminino, casados, com baixo grau de instrução, aposentados e com ren- 
da igual a dois salários mínimos.

A partir do perfil das estomias e dados clínicos desvelou-se predominância de estomia do tipo colostomia, definitiva, por câncer e confeccionada há mais de 3 anos. Tendo a HAS como comorbidades mais frequente, assim como, dependência total ou parcial para a realização do autocuidado cotidiano.

Estudos como esse podem auxiliar o profissional de saúde a planejar uma assistência individualizada para pessoas idosas com estomias considerando suas particularidades e fragilidades. Dessa forma, gerenciamento, implementações de atividades educativas e atendimentos clínicos podem ser qualificados após compreensão deste contexto.

\section{REFERÊNCIAS}

1. Alves JE. Envelhecimento populacional no Brasil e no mundo. Novas projeções da ONU. Rev. Longeviver [internet]. 2019jun [Acesso 22 set 2020]. Disponivel em: https://revistalongeviver.com.br/index. php/revistaportal/article/viewFile/787/842.

2. Ayaz-Akaya S. Overview of psychosocial problems in individuals with stoma: A review of literature. Int Wound J [Internet]. 2019feb [Acess 2020 sep 22];16(1):243-249. Available from: https://pubmed. ncbi.nlm.nih.gov/30392194/.

3. Barbosa MH, Poggetto MT, Barichello E, Cunha DF, Silva R, Alves PI, et al. Aspectos clínicos e epidemiológicos de estomizados intestinais de um município de Minas Gerais. Rev de Enfermagem e Atenção à Saúde [Internet]. 2014 [Acesso 22 set 2020];3(1):64-73. Disponivel em: http://seer.uftm.edu.br/revistaeletronica/index.php/enfer/article/view/931.

4. Carvalho SB, Freitas TO, Carvalho DJ. Percepção da pessoa com estoma acerca de sua sexualidade. UCSAL. 2019. [Acesso 22 set 2020]. Disponivel em: http://ri.ucsal.br:8080/jspui/handle/prefix/950.

5. Cascais AFM, Martini JG, Almeida PJ. O impacto da estomia no processo de viver humano [Internet]. 2007 [Acesso 27 out 2020]; 16(1):163-167. Disponivel em: https:/www.scielo.br/pdf/tce/ v16n1/a21v16n1.pdf.

6. Cerqueira LC, Cacholi SB, Nascimento VS, Koeppe GO, Torres VP, Oliveira PP. Caracterização clínica e sociodemográfica de pessoas estomizadas atendidas em um centro de referência. Rev. Rene. 2020. [Acesso 22 set 2020]; 21:e42145. Disponivel em: http://periodicos. ufc.br/rene/article/view/42145.

7. Costa AT da, Santana PPC, Teixeira PA, Santo FH do E, Flach DMA de $M$, Andrade $M$. Evidências científicas de enfermagem sobre idosos estomizados. REAID [Internet].2019abr [Acesso 22 set 2020];79(17). Disponivel em: https://revistaenfermagematual.com.br/index.php/ revista/article/view/336.

8. Macêdo ML, Cavalcante VM, Coelho MM, Ramos SL, Correia DL, Menezes TA, et al. Percepções de pacientes estomizados com câncer colorretal acerca da qualidade de vida. Rev. Rene. 2020 [Internet] 2020Jul [Acesso 22 set 2020];21:e43946. Disponível em: http://periodicos.ufc.br/rene/article/view/43946/161806.

9. Maciel DBV, Santos MLSC dos, Souza NVD de O, Fuly P do SC, Camacho ACLF, Soares HPL. Qualidade de vida de pessoas com Estomias intestinais definitivas: Uma revisão integrativa [Internet]. 2019 [Acesso 27 de out 2020]; 86(24) Disponível em: https://revistaenfermagematual.com/index.php/revista/article/view/109.

10. Malta DC, Araújo Andrade SS, Oliveira TP, Moura L, Prado RR, Souza MF. Probabilidade de morte prematura por doenças crônicas não transmissíveis, Brasil e regiões, projeções para 2025. Rev. bras. epidemiol. [Internet]. 2019 [Acesso 22 set 2020]; 22:e190030. Di- sponível em: https://www.scielo.br/pdf/rbepid/v22/1980-5497rbepid-22-e190030.pdf.

11. Mauricio VC, Oliveira Souza NV, Luz Lisboa MT. O sentido do trabalho para o ser estomizado [Internet]. 2014 jul/set [Acesso 22 set 2020];23(3):656-664. Disponivel em: https://www.scielo.br/pdf/tce/ v23n3/pt_0104-0707-tce-23-03-00656.pdf.

12. Moraes JT, Assunção RS, Sá FS, Lessa ER, Corrêa LS. Perfil de pessoas estomizadas de uma região de saúde mineira. Rev. Enferm Foco [Internet]. 2016 agosto [Acesso 22 set 2020];7(2):22-26. Disponível em: https://pdfs.semanticscholar.org/3fee/015319e14e9fb6d436804b7a2dbcb421a989.pdf.

13. Nicolato FV, Couto AM, Castro EA. Capacidade de autocuidado de idosos atendidos pela consulta de enfermagem na atenção secundária à saúde. Rev. Enferm Cent. O. Min. [Internet]. 2016 [Acesso 22 set 2020]; 6(2):2199-2211. Disponivel em: http:/www.seer.ufsj. edu.br/index.php/recom/article/view/1016.

14. Bonill-Nieves C de las, Celdrán-Mañas M, Hueso-Montoro C, Morales-Asencio JM, Rivas-Marín C, Fernández-Gallego MC. Living with digestive stomas: strategies to cope with the new bodily reality. Rev. Latino-Am. Enfermagem [Internet]. 2014jun [Acess 2020 sep 22];22(3):394-400. Available from: https:/www.scielo.br/pdf/rlae/ v22n3/0104-1169-rlae-22-03-00394.pdf.

15. Santos FS, Vicente NG, Bracarense CF, Dal-Poggeto MT, Goulart BF, Rodrigues LR. Percepção dos cônjuges de pessoas com estomia intestinal sobre a sexualidade do casal. Rev. min. enferm [Internet]. 2019. [Acesso 22 set 2020]; 23:e-1217. Disponível em: http://www. dx.doi.org/10.5935/1415-2762.20190065.

16. Silva AT, Sena RM, Nascimento EG. Perfil de morbimortalidade por câncer em idosos no território nacional brasileiro. Periódicos UFAM [Internet].2020 [Acesso 22 set 2020];21(15):2176-9141.Disponivel em: https://www.periodicos.ufam.edu.br/index.php/BIUS/article/ view/8017.

17. Swan E. Helping families and carers to support a person with a stoma and dementia. British Journal of Nursing [Internet]. 2018 [Acess 2020 sep 22]; 27: S16-7. Available from: https:/www.magonlinelibrary.com/doi/abs/10.12968/bjon.2018.27.22.S16.

18. Veras RP, Oliveira M. Envelhecer no Brasil: a construção de um modelo de cuidado. Rev. Saúde Colet [Internet]. 2018jun [Acesso 22 set 2020]; 23(6): 1929-1936. Disponivel em: https://doi. org/10.1590/1413-81232018236.04722018.

19. Verweij NM, Bonhof CS, Schiphorst AH, Maas HA, Mols F, Pronk $A$, et al. Quality of life in elderly patients with an ostomy: A study from the population-based PROFILES registry. Colorectal Disease [Internet].2018 [Acess 2020 sep 22]; 20(4):92-102. Available from: https://doi.org/10.1111/codi.13989. 